\title{
Incidence and Clinical Outcomes of Clostridium difficile Infection after Treatment with Tuberculosis Medication
}

Yu Mi Lee*, Kyu Chan Huh*, Soon Man Yoon ${ }^{\dagger}$, Byung Ik Jang ${ }^{\ddagger}$, Jeong Eun Shin ${ }^{\S}$, Hoon Sup Koo*, Yunho Jung", Sae Hee Kim", Hee Seok Moon", Seung Woo Lee**, and Daejeon-Chungchung Intestinal Research Group

*Department of Internal Medicine, Konyang University College of Medicine, Daejeon, ${ }^{\dagger}$ Department of Internal Medicine, Chungbuk National University College of Medicine, Cheongju, ${ }^{\neq}$Department of Internal Medicine, Yeungnam University College of Medicine, Daegu, ${ }^{8}$ Department of Internal Medicine, Dankook University College of Medicine, "Department of Internal Medicine, Soonchunhyang University College of Medicine, Cheonan, "Department of Internal Medicine, Eulji University Hospital, "Department of Internal Medicine, Chungnam National University Hospital, and **Department of Internal Medicine, Daejeon St. Mary's Hospital, The Catholic University of Korea College of Medicine, Daejeon, Korea

Background/Aims: To determine the incidence and clinical characteristics of tuberculosis (TB) medication-associated Clostridium difficile infection. Methods: This multicenter study included patients from eight tertiary hospitals enrolled from 2008 to 2013. A retrospective analysis was conducted to identify the clinical features of $C$. difficile infection in patients who received TB medication. Results: $C$. difficile infection developed in 54 of the 19,080 patients prescribed TB medication, representing a total incidence of infection of 2.83 cases per 1,000 adults. Fifty-one of the 54 patients (94.4\%) were treated with rifampin. The patients were usually treated with oral metronidazole, which produced improvement in 47 of the 54 patients (87\%). Twenty-three patients clinically improved with continuous rifampin therapy for $C$. difficile infection. There were no significant differences in improvement between patients treated continuously $(n=21)$ and patients in whom treatment was discontinued $(n=26)$. Conclusions: The incidence of $C$. difficile infection after TB medication was not low considering the relatively low TB medication dosage compared to other antibiotics. It may not be always necessary to discontinue TB medication. Instead, decisions concerning discontinuation of TB medication should be based on TB status. (Gut Liver 2016;10:250-254)

Key Words: Enterocolitis, pseudomembranous; Clostridium difficile; Tuberculosis; Rifampin

\section{INTRODUCTION}

Clostridium difficile is a toxin-producing anaerobic bacterium responsible for antibiotic-associated colitis, and it is now the most common infectious cause of nosocomial diarrhea. ${ }^{1} \mathrm{C}$. difficile infection (CDI) presents various clinical features including asymptomatic carriage, mild diarrhea, toxic megacolon, or fulminant pseudomembranous colitis. ${ }^{2}$ There has been a progressive rise globally in the incidence, severity, and complications of CDI in recent years. CDI has re-emerged with apparent greater morbidity and mortality. ${ }^{3-5}$ These changes in CDI epidemiology, attributed in part to an emerging hypervirulent strain of $C$. difficile, have been documented in several studies worldwide. However, the incidence and clinical characteristics of CDI in patients receiving medications for tuberculosis (TB) remain unclear.

Traditionally recognized risk factors for CDI include hospitalization, advanced age, gastrointestinal surgery or procedures, and exposure to antibiotics. ${ }^{6}$ Among these risk factors, antibiotic exposure history is the most important. In a nationwide study of the incidence and clinical features of CDI in Korea, ${ }^{7}$ most patients (91.7\%) had received antibiotics prior to the diagnosis of CDI. Anti-TB drugs are not commonly associated with the occurrence of CDI. The association of rifampin (RFP) with pseudomembranous colitis was first reported in $1980,{ }^{8}$ and a few subsequent cases have been reported worldwide. In the after mentioned nationwide CDI study in Korea, 11 of 1,367 patients who had taken anti-TB medications including RFP developed CDI. $^{7}$

In the United States and Canada, the incidence of TB in 2012 was 3.6 per 100,000 and 4.6 per 100,000 individuals, respectively. The incidence reported in Korea was of 108 per 100,000. So, while the incidence of $\mathrm{CDI}$ after TB medication could be considered unimportant in Western nations, TB remains prevalent in

Correspondence to: Kyu Chan Huh

Department of Internal Medicine, Konyang University College of Medicine, 158 Gwanjeodong-ro, Seo-gu, Daejeon 302-718, Korea

Tel: +82-42-600-9370, Fax: +82-42-600-9090, E-mail: kchuh2020@hanmail.net

Received on November 6, 2014. Revised on February 11, 2015. Accepted on April 4, 2015. Published online August 11, 2015

pISSN 1976-2283 eISSN 2005-1212 http://dx.doi.org/10.5009/gnl14435

@ This is an Open Access article distributed under the terms of the Creative Commons Attribution Non-Commercial License (http://creativecommons.org/licenses/by-nc/4.0) which permits unrestricted non-commercial use, distribution, and reproduction in any medium, provided the original work is properly cited. 
Korea, a country where many patients are prescribed at least a 4-month regimen of anti-TB therapy.

Presently, we focused on the TB medication-associated CDI incidence in Korea from 2008 to 2013, analyzed the clinical features, and explored the association of CDI with RFP use in patients treated with TB medications.

\section{MATERIALS AND METHODS}

This multicenter study included patients from eight tertiary hospitals. Cases that met the criteria for CDI were enrolled from 2008 to 2013. Patients who had no recorded variables and those administered any antibiotics within 1 month from the start of CDI were excluded.

\section{Clinical features}

A retrospective analysis was conducted to identify the clinical features of CDI patients who received anti-TB medications. The clinical data collected were gender and age; length of hospitalization; underlying conditions including chronic liver disease, chronic viral hepatitis B and C, and liver cirrhosis; and medication history including the use of anti-TB medications. In addition, the initial treatment regimens prescribed for patients with CDI, whether or not to terminate the administration of anti-TB medications to treat $\mathrm{CDI}$, treatment failure rate, and recurrence rates were evaluated.

\section{Definition of CDI}

Diarrhea was defined as a bowel habit change with over three unformed stools per day for $>2$ days. ${ }^{10}$ The definition of CDI was admitted patients with documented diarrhea and any positive outcome (one or more) in the following diagnostic tests: (1) C. difficile toxin A and/or B via enzyme; (2) positive stool culture for $C$. difficile; and/or (3) typical endoscopic findings, such as multiple white or yellow plaques. ${ }^{7}$ The stool samples were tested with the VIDAS C. difficile toxin (A II or A/B; Bio-Merieux, Marcy l'Etoile, France) or Wampole $C$. difficile tox A/B II method (TechLab, Blacksberg, VA, USA). Stool culture for $C$. difficile was performed by inoculating stool samples into anaerobically reduced cycloserine-cefoxitin-fructose agar at $37^{\circ} \mathrm{C}$ under anaerobic conditions for 48 to 72 hours in each institute. The identification for suspected $C$. difficile was conducted through analysis via Gram stain, spore stain, and/or a biochemical assay using an ANA identification test kit (Bio-Merieux).

Improvement of CDI was defined as the resolution of diarrhea by day 6 after treatment. ${ }^{7}$ Recurrence of CDI was defined as the development of CDI after at least 8 weeks of symptom resolution. Complicated CDI was when toxic megacolon, colectomy, or death related to CDI resulted from septic shock or perforation that occurred within 30 days of diagnosis of $\mathrm{CDI}^{7}$

\section{Statistical analyses}

All data were analyzed using SPSS version 11.0 (SPSS Inc., Chicago, IL, USA). The chi-square test or Fisher exact test was used for analysis of categorical variables. Data are expressed as mean \pm standard deviation or as median and interquartile range (25th-75th percentile) as appropriate. A p-value $<0.05$ was considered significant.

\section{Ethics statement}

This study was approved by the Institutional Review Board of Konyang University Hospital (protocol number: 13-31). Also the Institutional Ethics Committee of each hospital approved the study protocol. Informed consent was waived by the board, and this study was also approved by the Clinical Research Information Service (protocol number: KCT 0000808).

\section{RESULTS}

Of the 19,080 patients diagnosed with TB from 2008 to 2013, 54 patients developed CDI after receiving TB medication, representing a total incidence rate of $\mathrm{CDI}$ after $\mathrm{TB}$ medication of 2.83 cases per 1,000 TB patients. The clinical features of the 54 patients (median age, $68.3 \pm 16$ years; range, 25 to 93 years) are listed in Table 1 . Thirty-five of the 54 patients (64.8\%) were diagnosed with pulmonary TB. Other patients were diagnosed brain TB, bone TB, and TB lymphadenitis. The comorbidity rate involving risk factors of CDI including gastrointestinal surgery and cancer chemotherapy was only 16.7\% $(n=6)$. However, when including immunosuppressive associations including malnutrition, steroid use, chronic liver disease, and diabetes mellitus, 50\% of patients had an underlying disease associated with CDI. Most of the patients (74.1\%) were treated at admission. The duration of hospitalization was $29.5 \pm 27.3$ days (range, 4 to 114 days). Diarrhea was the major clinical manifestation. Fifty-one of the 54 patients (94.4\%) complained of diarrhea, fever was noted in 10 patients, and abdominal pain occurred in 16 patients (29.6\%).

Most patients were treated with a combination regimen including RFP (94.4\%). The latency period of the development of CDI after the initiation of TB medication varied markedly from 3 to 167 days (mean, $41.8 \pm 35.0$ days). Leukocytosis was observed in 22 of the 54 patients (40.7\%) and creatinine elevation was observed in three patients (5.6\%). Severe complicated CDI was evident in three patients resulting in two cases of renal failure and one death caused by sepsis secondary to CDI. The mortality rate of CDI after TB medication was 1.9\%. Twenty-five patients received a colonoscopy or sigmoidoscopy. Various endoscopic findings showed the presence of elevated, yellowish creamcolored pseudomembrane (52.4\%), and also erosions, ulcers, and mucosal edema (42.9\%).

Clinical outcomes of CDI after TB medication according to 
Table 1. Clinical Characteristics of Patients with Clostridium difficile Infection after Receiving Tuberculosis Medication

\begin{tabular}{|c|c|}
\hline Characteristic & Value \\
\hline Age, yr & $68.3 \pm 16.0$ \\
\hline Male sex & $28(51.9)$ \\
\hline \multicolumn{2}{|l|}{ Comorbidities } \\
\hline Liver disease & $2(3.7)$ \\
\hline Malignancy & $3(5.6)$ \\
\hline Gastrointestinal surgery & $3(5.6)$ \\
\hline Immunosuppressive state & $1(1.9)$ \\
\hline Others & $18(33.3)$ \\
\hline Duration of hospital stay, days & $29.5 \pm 27.3$ \\
\hline Outpatients & $14(25.9)$ \\
\hline $3-29$ & $26(48.1)$ \\
\hline $30-89$ & $11(20.4)$ \\
\hline$\geq 90$ & $3(5.6)$ \\
\hline \multicolumn{2}{|l|}{ Symptoms } \\
\hline Fever & $10(18.5)$ \\
\hline Diarrhea & $51(94.4)$ \\
\hline Pain & 16 (29.6) \\
\hline Nausea & $2(3.7)$ \\
\hline Abdominal distension & $1(1.9)$ \\
\hline Complication & $3(5.6)$ \\
\hline $\mathrm{WBC} \geq 15,000$ cells $/ \mu \mathrm{L}$ & $22(40.7)$ \\
\hline $\mathrm{Cr} \geq 1.5$ times the premorbid level & $3(5.6)$ \\
\hline \multicolumn{2}{|l|}{ Anatomical distribution* } \\
\hline Rectum only & $4(7.4)$ \\
\hline From sigmoid to rectum & $11(20.4)$ \\
\hline From descending to rectum & $5(9.3)$ \\
\hline From transverse to rectum & 0 \\
\hline From ascending to rectum & $5(9.3)$ \\
\hline \multicolumn{2}{|l|}{ Endoscopic findings } \\
\hline Normal & $2(4.8)$ \\
\hline Cream colored pseudomembrane & $22(52.4)$ \\
\hline Erosion & $6(14.3)$ \\
\hline Ulcer & $1(2.4)$ \\
\hline Edema & $11(26.2)$ \\
\hline
\end{tabular}

Data are presented as mean \pm SD or number (\%).

WBC, white blood cells; Cr, creatinine.

*Twenty-five patients received colonoscopy or sigmoidoscopy.

treatment are summarized in Table 2. Patients were usually initially treated with oral metronidazole $(n=48,88.9 \%)$ with a treatment duration of $11.6 \pm 4.3$ days. Most of these patients improved ( $n=47,87 \%)$. Twenty-three patients (42.6\%) received RFP continuously during CDI treatment. Among the patients who improved, 21 continued TB medications during CDI treatment and 26 patients discontinued. There was no significant difference in improvement between the continued group and
Table 2. Clinical Outcomes of Clostridium difficile Infection after Tuberculosis Medication according to Treatment

\begin{tabular}{lc}
\multicolumn{1}{c}{ Variable } & Value \\
\hline First treatment regimen & $47(87.0)$ \\
Metronidazole, orally & $1(1.9)$ \\
Vancomycin, orally & $6(11.1)$ \\
Metronidazole, intravenously & $11.6 \pm 4.3$ \\
Treatment duration, days & $23(42.6)$ \\
Continue tuberculosis medication including rifampin \\
Continue including rifampin & $21(38.9)$ \\
Discontinue including rifampin & $7(13.0)$ \\
Discontinue only rifampin & $3(5.6)$ \\
Others & \\
Improvement & $47(87.0)$ \\
Improvement by first regimen & \\
Improvement by second regimen & \\
Failure & $5(9.3)$ \\
\hline
\end{tabular}

Data are presented as mean \pm SD or number $(\%)$.

*First regimen: metronidazole, orally; ${ }^{\dagger}$ Second regimen: vancomycin, orally, and metronidazole, intravenously.

the discontinued group (44.7\% vs 55.3\%, $\mathrm{p}=0.19$ ). Twenty-four patients discontinued RFP during CDI treatment; of these, 16 $(66.7 \%)$ did not retry any anti-TB medications and five patients (20.8\%) retried other regimens excluding RFP. Among these patients, there was no recurrence.

\section{DISCUSSION}

CDI is considered to be the main cause of bacterial infectious diarrhea in nosocomial settings. The incidence, severity, and mortality associated with CDI have increased steadily. Unfortunately, there is limited epidemiological data and information of CDI in Asia including in Korea. The incidence of CDI in Korea (2.7 cases per 1,000 in 2008) was reported for the first time in 2012 by the Korean Association for the Study of Intestinal Disease. ${ }^{7}$ A recent prospective surveillance study in Canada reported an incidence of CDI of 4.6 cases per 1,000 adult admissions between 2002 and 2007, ${ }^{11}$ and in eight European countries, 4.1 cases per 10,000 admissions in 2008. ${ }^{12}$ Presently, the total incidence of CDI after TB medication was 2.83 cases per 1,000 from 2008 to 2013. Anti-TB drugs have been a rare causative agent of CDI. But, in our data, the rate of involvement of anti-TB drugs was not relatively low. We overlooked CDI after TB medication because anti-TB medication use was low compared with other antibiotics that can lead to general CDI.

Almost any antibiotic can lead to CDI, but broad spectrum antibiotics with activity against enteric bacteria are the most frequent causative agents. ${ }^{13}$ The most important causative agent of CDI among the conventional anti-TB medications is unclear. 
Some studies have reported RFP-induced pseudomembranous colitis. ${ }^{14-20}$ RFP has a wide range of antibiotic activity compared to those of isoniazid and ethambutol, which do not significantly affect the large bowel flora. Considering the pathogenesis of CDI, RFP may perturb the intestinal bacterial flora. Boriello et al. ${ }^{15}$ reported on a CDI patient with prolonged RFP use who displayed no recurrence after treatment with other anti-TB medication and recurrence after RFP retreatment. Park et al. ${ }^{14}$ and Yim et al. ${ }^{16}$ discussed pseudomembranous colitis (PMC) patients with anti-TB treatment including RFP who improved after termination of the anti-TB medications and had no recurrence after retrying anti-TB agents excluding RFP. We did not statistically prove an association of RFP with CDI through statistical analysis. The best method to prove the association RFP with CDI would be by retrial with a single regimen of RFP and then to observe if there was recurrence of CDI. However, it could not be tried due to ethical problems. Nonetheless, presently there was no development of recurrence after non-retrial of any anti-TB medication or reuse of anti-Tb medication excluding RFP. This strongly suggests that RFP was the causative agent of CDI.

The identified risk factors for CDI include hospitalization, advanced age, gastrointestinal surgery or procedures, and antibiotic exposure. ${ }^{6}$ The median age of the patients was $68.3 \pm 16$ years (range, 25 to 93 years), similar to another case series studies that documented six cases of RFP-associated PMC. ${ }^{17}$ The mean age of the CDI patients was $61.6 \pm 15.3$ years (range, 19 to 96 years), ${ }^{7}$ which was not significantly different to that of CDI patients after anti-TB medication. Most patients complained of diarrhea, similar to another case series. ${ }^{17}$ Presently, only six patients (16.7\%) had gastrointestinal surgery/procedures or cancer chemotherapy. But, including immunosuppressive states like malnutrition, steroid use, chronic liver disease, and diabetes mellitus, 50\% of patients had an underlying disease associated with CDI. An association of RFP-induced PMC with moderate to severe liver dysfunction has been reported. ${ }^{8,18,19}$ However, we could not find an association with CDI after TB medication with liver dysfunctions when we checked the underlying disease and the laboratory results of patients. In our data, total chronic liver disease was present in two patients, and in laboratory results of the liver function test, six patients (16.7\%) had elevated aspartate aminotransferase and/or alanine transaminase more than twice that of normal at CDI diagnosis. The latency period to develop CDI after TB medication was of $41.8 \pm 35.0$ days, which is longer than usual for CDI. In a nationwide study, ${ }^{7}$ the mean duration of antibiotic usage prior to CDI was $16.0 \pm 18.4$ days (range, 1 to 276 days). These results were similar to those of other studies. ${ }^{14,16,18,20}$ RFP may have lower capacity to perturb the intestinal bacterial flora than do other antibiotics. ${ }^{18}$

In clinical definition of CDI by the Society for Healthcare Epidemiology of America and Infectious Disease Society of America, supportive clinical data include leukocytosis, serum creatinine level, complication including hypotension or shock, ileus, and megacolon. Leukocytosis was observed in 22 patients $(40.7 \%)$, creatinine elevation in three (5.6\%), and severe complication in three patients. Of the latter three cases, two were renal failure and one was death caused by sepsis secondary to CDI. Even though the overall condition of all patients was poor due to TB, CDI after TB medication may have mild to moderate clinical features.

Moreover, treatment outcomes are relatively good. Of the 54 patients with CDI after TB medication, 48 patients (88.9\%) were prescribed oral metronidazole initially, and 44 patients (91.7\%) improved. It was not lower than that of the usual CDI treatment outcome. In other studies, of 1,367 patients with CDI, 796 patients (94.1\%) improved with oral metronidazole. ${ }^{7}$ However, the mortality rate in our study was $1.9 \%$, which is higher than that of the usual CDI in Korea (0\% to $0.51 \%),{ }^{7,21}$ and similar to that in Western countries (1\% to $2.5 \%$ ). ${ }^{22}$ We assume the reason for this was the small sample size.

CDI is usually treated with oral metronidazole $500 \mathrm{mg}$, three times per day or with $250 \mathrm{mg}$, four times per day for 10 to 14 days. It is recommended to discontinue therapy with the inciting antimicrobial agents as soon as possible. ${ }^{6}$ If anti-TB medication is a causative agent of CDI, it could be better to discontinue anti-TB medication, especially RFP. In our study, 31 patients (57.4\%) stopped RFP administration and 23 patients (42.6\%) received RFP continuously throughout CDI treatment. However, there was no significantly different improvement between the continued group (44.7\%) and the discontinued group (55.3\%). Most of the cases of RFP-induced PMC were treated for discontinuation of anti-TB medications. However, it was recently reported in Korea that PMC induced by anti-TB agents can be treated with maintenance of the anti-TB medications during prescribed oral metronidazole and probiotics. ${ }^{23}$ In this case, the patients with uncompensated tuberculosis had higher deterioration risk if there was discontinuation of anti-TB medication. So the patients were treated with maintenance of the anti-TB medication and also with the addition of both oral metronidazole and probiotics. There was no significantly different improvement between the continued group and the discontinued group. TB is still prevalent in some areas including underdeveloped countries and the north-east area of Korea, and patients are commonly prescribed at least a 4-month regimen. RFP is one of the first-line anti-TB regimens, and risk of TB-treatment failure is higher without RFP. Change to a second-line anti-TB regimen necessitates more treatment periods and increased adverse effects. A first-line anti-TB regimen including RFP is more critical than a second-line anti-TB regimen regarding efficacy and safety. Therefore, we propose that it is not necessary to always discontinue anti-TB medication. It is better to decide on the discontinuation of anti-TB medication depending on the patient's condition according to TB status.

There were several limitations to our study. First there was a small sample size, even though the data was collected from 
eight tertiary hospitals. Second, only tertiary hospitals participated in the study, so the study subjects tended to be more severely ill than patients in other hospitals. Third, the study was conducted retrospectively by reviewing CDI cases in the eight centers. There might be a difference between the hospitals regarding suspicion of CDI and diagnostic tools of CDI including $\mathrm{CD}$ toxin assay methods. We need to perform prospective studies and to co-work with the Department of Pulmonology for consensus with respect to CDI suspicion. Fourth, we could not analyze difference between TB medication-associated CDI group and other antibiotics-associated CDI group. Study of the risk factors of TB medication-associated CDI is necessary.

In conclusion, the incidence of CDI after TB medication was not low considering the relatively low TB medication use, compared to that of other antibiotics. Attention should be paid to patients who complain of abdominal pain or diarrhea while taking TB medications, because of the possibility of a TB medicationassociated CDI. It may not always be necessary to discontinue anti-TB medication. It is better to decide whether discontinuation of anti-TB medication or not depending on TB status.

\section{CONFLICTS OF INTEREST}

No potential conflict of interest relevant to this article was reported.

\section{REFERENCES}

1. Khanna S, Pardi DS. The growing incidence and severity of Clostridium difficile infection in inpatient and outpatient settings. Expert Rev Gastroenterol Hepatol 2010;4:409-416.

2. Gweon TG, Lee KJ, Kang D, et al. A case of toxic megacolon caused by Clostridium difficile infection and treated with fecal microbiota transplantation. Gut Liver 2015;9:247-250.

3. Karas JA, Enoch DA, Aliyu SH. A review of mortality due to Clostridium difficile infection. J Infect 2010;61:1-8.

4. Burke KE, Lamont JT. Clostridium difficile infection: a worldwide disease. Gut Liver 2014;8:1-6.

5. Seo GS. Clostridium difficile infection: what's new? Intest Res 2013;11:1-13.

6. Cohen SH, Gerding DN, Johnson S, et al. Clinical practice guidelines for Clostridium difficile infection in adults: 2010 update by the society for healthcare epidemiology of America (SHEA) and the infectious diseases society of America (IDSA). Infect Control Hosp Epidemiol 2010;31:431-455.

7. Kim YS, Han DS, Kim YH, et al. Incidence and clinical features of
Clostridium difficile infection in Korea: a nationwide study. Epidemiol Infect 2013;141:189-194.

8. Klaui H, Leuenberger P. Pseudomembranous colitis due to rifampicin. Lancet 1981;2:1294.

9. World Health Organization. WHO statistical information system [Internet]. Geneva: World Health Organization; 2014 [cited 2014 Apr 2]. Available from: http://www.who.int/tb/country/data/profiles/en/.

10. Kyne L, Warny M, Qamar A, Kelly CP. Asymptomatic carriage of Clostridium difficile and serum levels of IgG antibody against toxin A. N Engl J Med 2000;342:390-397.

11. Gravel D, Miller M, Simor A, et al. Health care-associated Clostridium difficile infection in adults admitted to acute care hospitals in Canada: a Canadian Nosocomial Infection Surveillance Program Study. Clin Infect Dis 2009;48:568-576.

12. Bauer MP, Notermans DW, van Benthem BH, et al. Clostridium difficile infection in Europe: a hospital-based survey. Lancet 2011; 377:63-73.

13. Kelly CP, Pothoulakis C, LaMont JT. Clostridium difficile colitis. N Engl J Med 1994;330:257-262.

14. Park JY, Kim JS, Jeung SJ, Kim MS, Kim SC. A case of pseudomembranous colitis associated with rifampin. Korean J Intern Med 2004;19:261-265.

15. Boriello SP, Jones RH, Phillips I. Rifampicin-associated pseudomembranous colitis. Br Med J 1980;281:1180-1181.

16. Yim SY, Koo JS, Kim YJ, et al. Rifampin-induced pseudomembranous colitis with rectosigmoid sparing. Clin Endosc 2011;44:137139.

17. Jung SW, Jeon SW, Do BH, et al. Clinical aspects of rifampicinassociated pseudomembranous colitis. J Clin Gastroenterol 2007; 41:38-40.

18. Nakajima A, Yajima S, Shirakura T, et al. Rifampicin-associated pseudomembranous colitis. J Gastroenterol 2000;35:299-303.

19. Fournier G, Orgiazzi J, Lenoir B, Dechavanne M. Pseudomembranous colitis probably due to rifampicin. Lancet 1980;1:101.

20. Chen TC, Lu PL, Lin WR, Lin CY, Wu JY, Chen YH. Rifampin-associated pseudomembranous colitis. Am J Med Sci 2009;338:156158

21. Doh YS, Kim YS, Jung HJ, et al. Long-term clinical outcome of Clostridium difficile infection in hospitalized patients: a single center study. Intest Res 2014;12:299-305.

22. Schroeder MS. Clostridium difficile: associated diarrhea. Am Fam Physician 2005;71:921-928.

23. Woo ML, Cho JH, Kim JH, et al. Anti-tuberculosis agents induced pseudomembranous colitis treated with maintaining anti-tuberculosis drugs. Korean J Gastrointest Endosc 2009;38:47-51. 